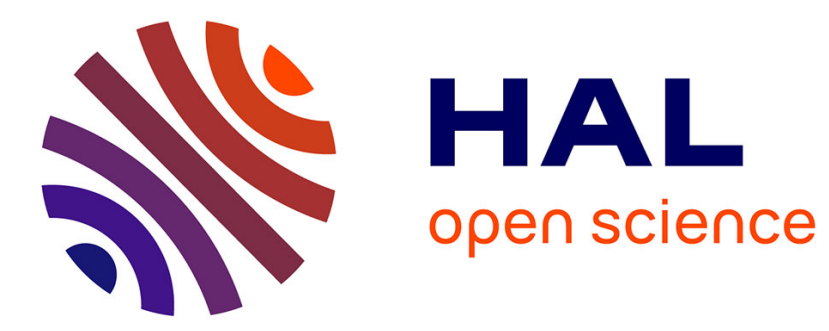

\title{
Le noël, miroir de la société. Du XVe au XIXe siècle Martijn Rus
}

\section{To cite this version:}

Martijn Rus. Le noël, miroir de la société. Du XVe au XIXe siècle. Neophilologus, 2009, 94 (2), pp.241-250. 10.1007/s11061-009-9182-8 . hal-00568383

\section{HAL Id: hal-00568383 \\ https://hal.science/hal-00568383}

Submitted on 23 Feb 2011

HAL is a multi-disciplinary open access archive for the deposit and dissemination of scientific research documents, whether they are published or not. The documents may come from teaching and research institutions in France or abroad, or from public or private research centers.
L'archive ouverte pluridisciplinaire HAL, est destinée au dépôt et à la diffusion de documents scientifiques de niveau recherche, publiés ou non, émanant des établissements d'enseignement et de recherche français ou étrangers, des laboratoires publics ou privés. 
Le noël, miroir de la société. Du XVe au XIXe siècle.

Abstract. The French "Noëls" (Christmas carols) were immensely popular from the end of the Middle Ages to the XIXth Century: they were sold in very large numbers (in fact: millions of copies). They often differ from the "Noël" in our days: they were not only sung, but also danced or played; they sometimes represented other scenes than that of the Nativity (such as the Annunciation), or even the (religious, literary, social or economic) reality of their time. It is this reflexion of reality in the "Noël" that I have studied in this article.

Key-words: Noël, Renaissance, Religious wars, XVIlth Century, Revolution.

Selon le Dictionnaire liturgique, historique et théorique de plain-chant de J. d'Ortigue, le noël est un "cantique spirituel, en langue vulgaire, en l'honneur de la naissance de Jésus-Christ" ; le Dictionnaire de la langue française d'E. Littré le définit comme un "cantique en langue vulgaire, ayant

ordinairement pour sujet la naissance de Jésus-Christ, que l'on chante à l'approche de la Noël" ; le Französisches Etymologisches Wörterbuch de W. von Wartburg, quant à lui, nous apprend qu'il s'agit d'un "cantique populaire chanté le jour de Noël"3; et citons, pour clore cet aperçu non-exhaustif (loin de là) des dictionnaires, le Petit Robert. le noël est une "chanson populaire dont le thème est Noël"

Ces définitions, comme toutes celles que l'on retrouve dans une multitude d'autres dictionnaires des siècles précédents, sont incomplètes cependant. Elles ne nous disent pas, d'abord, que le noël n'est pas toujours qu'un cantique, qu'une chanson: car il arrive qu'il prend une dimension nettement théâtrale, j'entends qu'il est joué par des personnages qui se produisent sur scène, comme c'est le cas de maints Mystères de l'Incarnation et de la Nativité, du Mystère de la Nativité de Barthélémy Aneau (1539), par exemple ${ }^{5}$. Ensuite, et c'est plus important, elles ne nous disent pas que le noël n'a pas toujours pour sujet exclusif la Nativité: d'autres scènes peuvent s'y rattacher, ou même la remplacer - comme celle de la fuite de Marie, Joseph et l'Enfant en Egypte, du massacre des Innocents, et l'on remonte parfois avant la naissance du Christ: à l'Annonciation et jusqu'à la tentation d'Eve. Voire, le noël a pu servir à tel autre but que celui dicté par la foi héritée du Moyen Age: plus d'une fois, dès son apparition dans l'imprimé, au XVe siècle ${ }^{6}$, jusqu'à son déclin au XIXe, il se trouve être lié à l'actualité, et ce, non seulement dans le domaine de la religion, mais dans celui de la culture, de l'histoire et de l'économie. S'y retrouvent, par exemple, les effets des guerres de religion, le souffle de la Renaissance, les échos de la vie des privilégiés qui vivent à la cour du roi, et, inversement, des démunis qui souffrent des lourdes taxes qui leur sont imposées, ainsi que les convictions athéistes des suppôts de la Révolution ${ }^{7}$. En sorte qu'il me semble permis de considérer le noël, tel noël, comme un miroir de la société, dans l'un ou l'autre de ses aspects, à un certain moment de son devenir. C'est cela, cette thèse, que je me propose d'illustrer dans le présent article, textes en main.

Avant que je m'engage dans ce périple, je constate que j'avais un terrain pratiquement vierge à déblayer. D'une part, en effet, les études consacrées aux noëls sont, la plupart du temps, d'inspiration éminemment religieuse: elles nous renseignent abondamment sur la foi naïve mais inébranlable des bergers et bergères qui viennent adorer l'Enfant nouveau-né, sur le bétail présent dans l'étable, les cadeaux qu'offrent les Rois Mages à l'Enfant, le rôle de Joseph (qui allume le feu, s'occupe de la marmite, apporte des langes), ou même sur la bûche qui flambe, jusqu'au XIXe siècle au moins, dans mainte maison en France lors de la vigile de Noël ${ }^{8}$ - mais elles passent généralement sous silence tout élément qui puisse nuire à l'image béate de la Nativité: les propos gaillards, voire obscènes, les

${ }^{1}$ d'Ortigue (1860, p. 919).

2 Littré (1869, p. 732b).

3 von Wartburg (1955, p. 38a).

${ }^{4}$ Petit Robert (1989, p. 1274b).

${ }^{5}$ Aneau (1539); cf. Poulaille (1942, pp. 117 ss.). Inversement, l'idée que les Mystères ont influencé le noëlcantique est généralement admise depuis l'étude exemplaire d'E. Mâle (1924, pp. 76 ss.)

${ }^{6}$ Le plus ancien recueil de noëls date de 1483 (S'ensuivent aucuns ditéz et chançons faites en l'honneur de la Nativité Jhesu Christ commençans par Noël); le premier auteur de noëls connu est Jehan Tisserant, prédicateur, mort en 1494.

${ }^{7}$ Constatons, pour éviter tout malentendu, que cette infiltration de l'actualité ne signifie point du tout que le noël "ancien style" cesse d'exister: il continue à vivre, à jouir d'une popularité immense au travers des siècles.

${ }^{8}$ Vloberg (1953, pp. 142 ss.). 
traits satirico-politiques, etc. D'autre part, les noëls ont servi à rassasier la faim de plusieurs musicologues, qui, bien évidemment, ne se soucient guère des textes tels qu'en eux-mêmes - mais qui ont établi, par exemple, que des compositeurs de renom s'y sont essayés, comme Jannequin et Despréz, et que le chant, ou mieux l'air, la mélodie qu'emprunte le noël n'est souvent pas en correspondance avec le thème religieux du texte: il peut s'agir de l'air d'une chanson de danse populaire, d'une chanson d'amour ("Nicolas va voir Jeanne"; "Ma mère, mariez-mol”), d'une chanson gaillarde ("Hau Margot, liève la cuysse") ${ }^{10}$, d'une chanson à boire ("Le vin, le vin, le vin, le vin") ${ }^{11}$ ou, pire encore, d'une danse du ventre ${ }^{12}$.

Mon but est d'étudier le noël en tant que texte - indépendamment de toute interprétation d'extraction religieuse ou musicale, dans la perspective que j'ai dite. Or, il convient d'abord de constater, après $P$. Zumthor, que le noël (traditionnel) reproduit, en principe, des formes poétiques lettrées religieuses ou profanes des XIIle, XIVe et XVe siècles, ayant trait à la Nativité ${ }^{13}$ - formes et Histoire familières au commun des fidèles ${ }^{14}$. Ensuite, le langage qu'on rencontre dans la plupart des noëls, relève du "registre oral" - on a dit "populaire" - dont voici un spécimen: "Les bergers et bergerettes / Gardent leurs brebis aux champs, / Ouyrent la voix céleste; / Ilz s'endormirent au chant. / Guillot dormait, / Et si soufflait, / Gorget et Jaquemette, / Et Robinet / Ron ron ronflait / En songeant mains chousettes. // Par derriere une bayette / Le loup vint a ce matin; / Trip, trip, trip, faict la chevrette, / Vou, vou, vou, faict le mastin, / Betz, betz, betz, le chevreton, / Maiz, maiz, les brebiettes, / Pru, pru, du pied faict le mouton [...]" $]^{\prime 15}$. En plus, le caractère oral de ces poèmes est teinté parfois d'un réalisme grossier qui remonte, comme on sait, à une ancienne tradition gauloise: "[Dialogue de bergers] Que t'es-tu levé faire / Pastoureaulx à minuit? / Qué rage as-tu à braire / Si fort Naulet [Noël], Naulet, / Es-tou pas accouché / Ta femme cette nuit? / As-tu tué ta truie / Ta truie ou ton goret?"16. "[Rogelin] Et toy Ruben, ton chapperon affuble / Vent de l'aulnay [aulnaie] souffle au cul [de] la bergiere. // [Raguel] Bergiere Rachel prens le / Si dancerons ung branle, / Mais garde sur la glace / Tomber, car il verglace, / Abas: debout: trop les jambes tu haulses, / Cache ton cul, car tu n'as point de chaulses. // [Rachel] Couvre moy doncq' tombée à la renverse, / Gentil bergier, si j'ay la cuisse haulte"17.

Pourtant, plus d'un noëliste aspire à une forme de langage plus sophistiquée, plus "poétique": "Pastoureaux en veille / Avec leurs troupeaux / Les vignes sont belles / Et tous les blez beaux. / Ont veu extincelles / Reluisans flambeaux / Nouel! Nouel! // Ont veu extincelles / Reluisans flambeaux / Les vignes sont belles / Et tous les blez beaux, / Ouyrent nouvelles / Et propos moraux / Nouel! Nouel! // Ouyrent nouvelles / Et propos moraux / Les vignes sont belles / Et tous les blez beaux [...]" ${ }^{18}$. II arrive même, à l'aube des temps dits modernes, que tel poète s'engage dans le chemin, à la mode alors, de ceux qu'on a coutume d'appeler les "Grands Rhétoriqueurs": "Plaisir n'est pas que vivre en desconfort, Confortez-vous, gens de noble valeur, / L'heur de Jésus combatra tout malheur, / Malheureux est qui n'espère confort. / Noël! [...]"19. "C'est l'ange messaiger saige, / C'est l'ange messaige, / II dict, qu'ennuyct a la mynuict / Le filz Dieu voulut naistre: / Toute la nuyct point ne m'a nuict / Veillant par ce champ estre; / Laisson parc champestre paistre, / Laisson parc champestre; /

\footnotetext{
${ }^{9}$ Bachelin (1927, p. 143).

${ }^{10} I d .$, p. 138.

${ }^{11}$ Poulaille (1950, p. 140).

${ }^{12}$ Bachelin, op.cit., p. 143.

${ }^{13}$ Zumthor (1984, p. 96).

${ }^{14}$ D'où, sans doute, l'immense popularité des noëls, même avant l'invention de l'imprimerie, témoin les Crieries de Paris de Guillaume de la Villeneuve (fin du XIIIe siècle): "[On vend dans les rues de Paris] Noël! Noël! A moult granz cris", dans: Barbazan (1976, p. 282). Cette popularité se perpétuera au cours des siècles suivants: une légion de colporteurs les vendent, à bas prix, à des millions d'exemplaires - alors que les autres best-sellers de ces temps n'atteignaient, sauf exception, qu'à un tirage de 1000 à 1500 exemplaires, chiffre qui se stabilisera jusqu'au XIXe siècle (cf. Fèbvre et Martin, 1971, pp. 307-313).

${ }^{15}$ Noël! Noël! Noël! Chantons, dans: La Fleur des Noëls, Lyon, 1535, éd. Poulaille (1950, pp. 90 ss.).

${ }^{16}$ Lucas Le Moigne (fin du XVe siècle), dans: $i d$. (1942, p. 300).

${ }^{17}$ Aneau (1539), Chant pastoral, en forme de Dialogue, a trois bergiers, et une bergiere, contenant

l'Annonciation de l'Ange aux pasteurs, p. B3.

${ }^{18}$ Nicolas Martin (1498-1566), Nouel! Nouel!, dans: Poulaille, (1942, pp. 420 ss.).

${ }^{19}$ Jehan Daniel (première moitié du XVIe siècle), dans: ibid., pp. 336-337.
} 
Allon grand alleure, a l'heure, / Allon grand alleure [...] // Dieu gard la commere, mere [...] // Le beuf d'une lesche, leiche / Le beuf si le leiche; / D'aultre part l'alaine l'asne, / D'aultre part l'alaine [...] // Une chansonnete nete, / Une chansonnete; / Tant que l'air resonne, et sonne, / Tant que l'air resonne" ${ }^{20}$.

Ces citations prouvent que certains auteurs de noëls sont au courant de ce qui fait l'actualité, en leur temps, en matière poétique - actualité, ou mode, à laquelle ils tâchent de se conformer dans leurs propres productions. Mais ce n'est pas tout: car plus d'un noël se trouve être marqué par ce que j'ai appelé ci-dessus le "souffle de la Renaissance". Ainsi, dans bien des poèmes, notamment dans les scènes pastorales, les bergers et bergères ne s'appellent plus, comme au bon vieux temps, Robin, Guillot, Colas, Margot ou Guillemette, mais portent des noms qui remontent à l'histoire de l'Antiquité, comme Alaïs, Corydon, Daphne, Philandre ou Tircis. Mieux, on nous fait remarquer parfois que ces bergers ne sont point des illettrés: "L'un avoit leu en Joel / Et l'autre en Ezechiel / Que le doux Emmanuel / A Noël / Descendroit du saint concierge / Et nasquiroit d'une vierge" ${ }^{\prime 21}$.

Ce sont là des témoins mineurs de l'"esprit du temps", peut-être même de simples opérations cosmétiques effectuées sur la surface d'un texte, de tel texte, destinées à doter celui-ci d'un air moderne, à renouer le texte avec une inspiration "renaissante": l'essentiel est ailleurs. II réside plutôt dans le fait qu'on voit apparaître, dès le second tiers du XVle siècle, des noëls, dûs à des auteurs dont la renommée retentit jusque dans les manuels et florilèges de nos jours, qui s'opposent fondamentalement aux poèmes traditionnels. Et ce, parce que leur langage n'a plus rien à faire avec le "registre oral" (ni non plus, conséquemment, avec la tendance gaillarde ou gauloise qui s'y lie plus d'une fois, comme j'ai dit): il relève plutôt du domaine des lettres, du registre de l'écriture, poétique en l'occurrence, au sens que Roland Barthes a donné à ce mot, par un certain raffinement du mouvement rythmique qui s'y retrouve, la variété des effets de coupe, l'élégance de telle tournure, d'un vers bien frappé, en un mot par le travail du poète sur la forme du sens: les sons, les mots, la texture grammaticale (travail-topos, comme on sait, de la doctrine des poètes de la Pléiade).

Qu'on en juge par les morceaux suivants: "[Dialogue d'une pastourelle et d'un berger] Te souvient-il plus du Prophète, / Qui nous dit cas de si haut fait, / Que d'une Pucelle parfaite / Naîtrait un Enfant tout parfait? / L'effet / Est fait / La belle / Pucelle / A un fils du Ciel avoué [...]"22; "[Le berger Sophron, id est le Prudent, s'adresse à Marie] Moy, qui pour tous dois faire la harangue, / Confesser veux n'avoir force ny langue, / Ny nul sçavoir, pour vous remercier. / Rien ne pouvons, fors nous humilier / Devant l'enfant, ou la divinité / Veult habiter par son humilité; / Offrant tout ce qu'en nous le Pere a mys, / Amys d'amys, ennemys d'ennemys. / Vivre et mourir voulons en te servant; / Vivre sans toy estimons moins que vent [...]" ${ }^{23}$; "La Terre au Ciel, l'Homme à la Déité, / Sont assemblez d'un nouveau mariage. / Dieu prenant corps, sans faire au corps outrage, / Naist aujourd'huy de la virginité. // La Vierge rend à la Divinité / Son sainct depost, dont le Monde est l'ouvrage, / Mais aujourd'huy il a fait d'avantage, / S'estant vestu de nostre humanité! [... $]^{24}$.

Enfin, il est frappant de constater que ces noëls "renaissants" ne présentent pas de références à la mythologie classique (auxquelles je m'attendais): celles-ci ne se rencontrent que plus tard, au XVIIe siècle ("Et toy chien / Stygien / Qui tempêtes / De trois têtes / En ces lieux / Ennuyeux / Que t'en semble? [...]"25) et au XVIIIle siècle ("Par la permission du Ciel / Le maudit cerbère / S'échappant par la lucarne / Ou par la chatière / Dans Eden il s'en allait / Ou dame Eve demeurait / Tra la la la la la la, / Porter la misère" ${ }^{\prime 26}$ ).

Mais le XVle siècle n'est pas seulement celui de la Renaissance: il est profondément marqué aussi par une crise religieuse, provoquée par la révolte de Luther, qui finit par dégénérer en guerre civile, favorisée d'ailleurs par des raisons d'ordre politique (les tendances démocratiques des calvinistes, le

\footnotetext{
${ }^{20}$ Aneau, (1539), Noël branlant, p. B4.

21 Samson Bédouin (- 1563), dans: Poulaille (1942, p. 357).

${ }^{22}$ Clément Marot (1987), Chanson du jour de Noël, pp. 232-233.

${ }^{23}$ Marguerite de Navarre (s.d.), Bergerie, pp. 70-71.

${ }^{24}$ du Bellay (1934), Sonnet du jour de Noël, pp. 262-263.

${ }^{25}$ Anonyme, De la nuit de Noël. De la victoire gaignée par Notre-Seigneur sur la mort et l'enfer, dans: Poulaille (1950), p. 410.

${ }^{26}$ Anonyme, La Chute de notre premier père, François Gauthier, imprimeur, 1720, dans: ibid. p. 517.
} 
problème de la différence de religion entre le roi et une partie de ses sujets, cause de la décadence du prestige et du pouvoir royals). Or, maint noël témoigne, à sa manière, de cette crise. Je donne la parole d'abord aux poètes de l'ancienne foi, catholique - et l'on verra que leurs noëls ont bien souvent l'air d'un pamphlet politico-religieux: "Chantons Noël, priant de cueur non fainct / Et les vilains qui par tout l'ont semée [id est l'hérésie], / Que plus ne soit hérésie estimée [...]" "27; "[La Vierge Marie dit] Je suis amye de tous les bons chréstiens / Je suis haïe de tous les Luthériens / Je suis servie de tous vrays pénitens" ${ }^{28}$; "Les bons pasteurs convient hanter / Dont debvrions Noël chanter / Et les roys sans nous exempter / De bien faire / Et retraire / Luthériens qui veuillent braire / Et soubs la cheminée prescher / Contre le roy que tenons cher" ${ }^{29}$; "Donnons gloire au Sauveur Jésus, / Des huguenots il n'y aura plus: / Car le Roy n'en souffrira pas, Alleluya. // Quittez l'hérésie de Calvin / Qui n'a jamais fait aucun bien: / Et le seigneur vous bénira, Alleluya" ${ }^{30}$; "Te prions à genoux, / Que nous puissions vivre tranquilles, / Partout aux champs et dans les villes, / Obéissant au Roy. / Maintiens notre foi catholique / Et extermine l'hérétique / Qui méprise ta loi" ${ }^{31}$.

Quant à l'autre, l'hétérodoxe (nommé "hérétique", "Iuthérien" ou "huguenot" dans les poèmes susdits), il convient de constater qu'assez peu de noëls portent une signature protestante - et encore ne se distinguent-ils guère des noëls catholiques traditionnels. Ainsi: "Ame douillette esveille-toy! / Chante Noël joyeusement / D'un coeur gaillard sans nul esmoy / Et contemple dévotement / Le doulx aigneau / Et chante Nau [Noël] "32; "Un enfantelet nous est né aujourd'hui, Fils d'une Vierge, il est doux et beau; il fera votre bonheur et votre joie. C'est le Seigneur Christ notre Dieu, lui qui veut vous délivrer de tout mal; il vient pour être votre sauveur, pour vous rendre purs de tout péché $[\ldots]^{33}$. Mais il y a une exception à cette règle, et c'est le Chansonnier huguenot qui nous la livre: 'L'on sonne une cloche / Dix ou douze coups / Le peuple s'approche / Se met à genoux / Le prestre se vest / Harri, Harri l'âsne, le prestre se vest / Harri bourriquet. // [...] // Du sainct Evangile / En prend quelque endroit / Qu'il coupe et mutile [...] // Un morceau de pâte [l'hostie] / II fait adorer / Le rompt de sa patte / Pour le dévorer / Le gourmand qu'il est [... $]^{34}$.

Je passe à une autre facette du noël-miroir, celle de la représentation du contexte vécu par le commun des mortels - contexte socio-économique (j'entends la communauté à laquelle il appartient: ville, village, bourg, bourgade, paroisse, ou métier), et géographique (les "lieux de mémoire" que la communauté entretient: cathédrale, église, auberge ou taverne). L'on verra que les références à ce contexte ne sont pas toujours dénuées de fierté locale ou, tout au plus, régionale (car l'horizon de l'homme du Moyen Age ne s'étend guère, la plupart du temps, au-delà des murailles de sa ville, des frontières de son village ou bourg, la lisière d'une forêt), voire elles frisent parfois ce qu'on appelle aujourd'hui le message publicitaire ${ }^{35}$. Voici quelques extraits, choisis parmi une foule d'autres, en guise d'illustration: "Tous les bourgeois de Chastres / Et de Mont-le-Héry, / Menèrent grande joie / Cette journée-cy / Que nasquit Jésus-Christ [...] // [Les bergers] Laissèrent leurs troupeaux / Paissant parmy les champs, / Prirent tous leurs chalumeaux / Et droit à Saint Clément / Vinrent dansant,

\footnotetext{
${ }^{27}$ Jehan Daniel, Chansons nouvelles de Nouel / Composées tout de nouvel / Esquelles verrez les praticques / De confondre les héréticques (vers 1555), dans: Poulaille (1942, p. 342).

${ }^{28}$ Anonyme, Je suis Marie, le Vaisseau précieux, Bible des Noëls de Sébastien Molin, vers 1555, dans: ibid., p. 521.

${ }^{29}$ Denis Gaigniot (?), Nous debvrions Noël chanter, s.d., dans: ibid., p. 550.

${ }^{30}$ Anonyme, Sur la conversion des huguenots, noël poitevin, s.d., dans: ibid., p. 213.

${ }^{31}$ Anonyme, Nous te requerrons à mains jointes, noël bressan, 1591, dans: ibid., p. 583.

${ }^{32}$ Mathieu Malingre, Ame douillette esveille-toy!, Belles et bonnes chansons que les chrétiens peuvent chanter en grande affection de coeur, 1533, dans: ibid., p. 378.

${ }^{33}$ Martin Luther (1483-1546), Je viens du ciel élevé, trad. G. Albin, Ballades et chants populaires de l'Allemagne, Paris, 1841, dans: ibid., p. 360.

${ }^{34}$ Anonyme, Noël de la description. Forme et manière de dire la messe, 1564, Chansonnier huguenot, dans: ibid., pp. 541-543.

${ }^{35}$ Il est intéressant de faire remarquer, dans cette perspective, que les grands du royaume aimaient à se faire représenter, dans l'iconographie, en dévotion aux pieds de la Vierge et de l'Enfant, voire en figure de Mages défilé qui s'achève sur une estampe gravée par Léonard Gaultier pour un almanach de 1611, où les princes et princesses de la Maison de France figurent, et, c'est significatif, me semble-t-il, avec moins de regard pour l'Enfant que pour l'artiste (cf. Vloberg, 1953, pp. 21-28).
} 
chantant [...] // Puis ceux de Sainct Germain / Tous en procession, / Partirent de bon matin / Pour trouver l'enfançon [...] // Les pasteurs des Bruyères / N'estoient pas endormis, / Sortirent des tanières [...] // Puis eussiez vu venir / Tous ceux de Sainct Yon / Et ceux de Brétigny [...]"36; "Du jardin de la France / II vint des pastoureaux, / Que pour leur différence / L'on nomme Tourangeaux, / Présenter à la Reine / Des beaux fruits de Touraine, / Et des draps les plus fins / De tous les magasins [...] // Saint Saturnin ensuite / Donna de son trésor, / Une étoffe d'élite / De brocart de fin or, / Sa frange et sa doublure / D'une riche parure, / Tous les plus beaux atours / Qui fussent dedans Tours. // Les marchands de soierie / De Saint-Pierre Puellier / Sont venus voir Marie, / Et son fils supplier, / D'un coeur humble et sincère, / De recevoir leur chère, / Et ne refusèrent pas / De leurs beaux taffetas [...]"37; "S'il eust pleu au bon Dieu / de prendre chair humaine / Au Mans en quelque lieu / les pastoureaulx du Maine / fussent venuz de Maienne / de Laval, de Sablé, / c'est chose bien certaine, / du Loir et de La Ferté [...] // De Lassay et Goron / De Sillé et Charnie / De tout le pays d'Esvron / De Champaigne et Conlye / N'eussent failly mye [...]"38; "Pâris, cours à la Halle, / Va chercher du poisson; / Monte sur la cavale / Du compère Buisson; / Prends des huitr'à l'écaille, / Des soles, du saumon [...] // Pour de l'épicerie / Hérard en fournira; / Viande de boucherie, / Chez Dereims en aura; / De la rôtisserie / Cotteret en donnera [...]"39; "[Joseph] Auriez-vous, monsieur l'hôte, / Maître du GrandDauphin, / Quelque grenier ou grotte, / Ou quelque petit coin? // [...] // Voyons la Rose-Rouge / Madame de céans, / Auriez-vous quelque bouge / Pour de petites gens? // [...] // Monsieur des TroisCouronnes, / Avez-vous logement / Chez vous pour deux personnes [...] // Et vous, monsieur le maître / Des Trois-Petits-Paniers, / Pouvez-vous point nous mettre / Dans un coin du grenier? [...]" ; "Si le chemin de cette sainte étable / Nous semble trop difficile et trop long, / Allons nous rendre dans la cathédrale, / A Saint-Aignan ou à Saint-Pierre-en-Pont. / Ou bien, sans sortir de notre paroisse, / Allons à l'église de Saint-Victor [...." $]^{41}$.

Ensuite, dans le prolongement de ce qui précède, le noël reflète aussi les circonstances réelles de la vie des humbles, misérable, hélas, la plupart du temps: "Marie, sainte-mère, / Nous prions, écoutenous: / Fais tant que Dieu le père / Emplisse les bennes [seaux des vendangeurs] ou les benons [paniers des boulangers]. / Noël, Noël, etc. // Délivre-nous de la griffe / De ces usuriers / Et de la fausse trappe / Des aventuriers [corps militaires]. / Noël, Noël, etc. // Venge-nous de la tromperie / Que nous font les sergents [huissiers] [...] // Fais abaisser la taille [les impôts] / Qui nous tuera, je crois [...]" $]^{\prime 2}$; “[...] moy je n'ay mie / Pour calmer ma faim. / Bon roy, je vous prie, / Baillez-moi du pein $[\ldots]^{\text {"33 }}$; "Nous te requerrons à mains jointes / Vouloir ouïr mes griefves plaintes / Nous, pauvres pastoureaux / De toutes parts, on nous sacage / On nous détruit, on nous ravage / En brebis et agneaux. // Le soldat tous les jours, sans cesse / En nos casettes [cabanes] nous oppresse / Pille et emporte tout / II nous compresse [accable], il nous rançonne / A son départ, souvent nous donne / Encore un meschant coup [... $]^{34}$.

Mais, a contrario, le noël chante parfois aussi la joie de ces mêmes déshérités à l'occasion de la naissance du Christ - car ceux-ci savent faire la fête, en dépit de leur vie misérable, à tel momentsommet de l'année liturgique (dont le carnaval est le plus célèbre): "Voici le temps compassé / Que dans ce lieu / Faut mener joyeuse vie; / Et chanter tantirelilonfa, / Pour chasser mélancolie [...] // Sus, c'est assez caqueté / Verse du vin, / Chambrière qu'on se haste / La voyez à ce pasté [...] // Garçon emply-moy ma tasse, / Rebeuvons, / Voicy je vais boire à toy [...] // Ce vin n'est pas trop piret [mal] /

\footnotetext{
${ }^{36}$ Y.L. Crestot, Tous les bourgeois de Chastres, dans: Grans Noelz nouvellement imprimés à Paris pour Jean Bonfons, vers 1520, éd. de Smidt (1932, pp. 146ss) [je souligne].

${ }^{37}$ Anonyme, Pastourelle des paroisses de Tours (seconde moitié du XVIIe siècle), éd.cit., p. 215 [je souligne].

${ }^{38}$ Samson Bédouin, S'il eust pleu au bon Dieu, s.d., dans: Poulaille (1942, p. 358 [je souligne]).

${ }^{39}$ Anonyme, Voisin, viens à la Crèche (ca. 1650), dans: id., (1950) p. 390 [je souligne].

${ }^{40}$ Anonyme, Nous voici dans la ville, dans: la Grande Bible renouvellée de Noëls nouveaux, à Troyes, chez Jean-Antoine Garnier, s.d., éd. de Smidt, éd.cit., p. 245 [je souligne].

${ }^{41}$ Anonyme, Noël de la paroisse Saint-Victor d'Orléans, dans: Belle Bible de Tours, 1688, éd.cit., p. 168 [je souligne].

42 Anonyme, Laissez chômer les pioches, noël bressan, 1536-1545, dans: Poulaille, (1942, p. 527).

${ }^{43}$ F. Lozat, Le Noël de Povret, 1596, dans: ibid., p. 545.

${ }^{44}$ Anonyme, Nous te requerrons à mains jointes, cité supra (note 31 ).
} 
Veu la saison, /ll est d'assez bonne sêve, / Sire Roy, beuvez à nous / Deux ou trois coups, / Afin de tremper la fève / Ce chapon tantirelonfa $[\ldots]^{34}$.

Ce n'est pas tout. En même temps, en effet, paraît un autre groupe de noëls qui, eux, s'occupent de la vie des grands, de ceux et de celles qui font partie du beau monde d'alors: ce sont les noëls dits de cour, qui enregistrent à leur manière, essentiellement satirique, les moindres bruits à leur sujet - ils constituent, si l'on veut, une sorte de presse à scandale avant la lettre. Ces noëls, comme bien on pense, ont été mal aimés de la critique (aussi sont-ils quasi introuvables): il s'agirait d'une "littérature de bidet", d'un ensemble de "boîtes à ordures", de "tissus de sottises, de saletés, de vilenies", etc. ${ }^{46}$ En voici quelques spécimens: "Or, nous dites , la Baume ${ }^{47}$, / Pourquoi venir loger / Près l'hôtel de Vendôme / Pour nous faire enrager? / - J'étais par trop connue / Au faubourg Saint-Germain; / Les enfants dans la rue / Crioient: Ah! La p[utain]!" " "Nos femmes et les coquettes / S'en iront tour à tour / Ecouter les fleurettes / Des messieurs de la cour; / Pour surcroît de misère, / Après nos biens perdus, / Elles nous feront pères / D'un millier d'inconnus"49; "D'une maigre duchesse / Quatre jeunes amants / Vinrent de leur maîtresse / Offrir les beaux présents [...] / Joseph dit au poupon, / Don, don, / Ne touchez pas cela, / La, la / Vous prendrez la vérole [...] // Saint Quentin la punaise / Avec son vieil amant / Etant mal à son aise / Et vivant pauvrement / Dit au divin poupon: / Trouvez bon, je vous prie, / Qu'au bord [et] sans façon, / Don, don, / J'aille faire cela, / La, la, / Pour y gagner ma vie. // La chose est malaisée, / Lui répondit l'enfant, / Vous estes trop usée / Par vos avortements" ${ }^{, 50}$.

Je ne suis pas encore au bout de mon périple: car il existe aussi des noëls qui reflètent l'idéologie de la Révolution, des noëls sans culottes, où les Rois Mages sont remplacés par des concitoyens célèbres alors, et où la Vierge et l'Enfant cèdent la place à la Liberté et ses entités soeurs: "A bas les rois! A bas leur caste meurtrière / A bas les rois! [...] // Des potentats, / Ces cruels oppresseurs du monde, / Des potentats, / Qui compterait les attentats / Sans éprouver l'horreur profonde / Qu'inspire cette race immonde / Des potentats?"51; "Ah! Que la loi nouvelle / A de douceur! / D'un saint amour pour elle / brûle mon coeur: / La liberté, l'égalité des hommes / Dans le siècle où nous sommes / Vont faire le bonheur. // Despotisme exécrable, / monstre insensé; / ton trône abominable / est renversé [...]" ${ }^{52}$; "Dieu se fait sans culotte / Pour nous ouvrir les cieux./ D'une Vierge dévote, / II naît en ces bas lieux [...] // Législateurs et rois, / A l'exemple des mages, / Viennent avec empressement / Aux pieds / De ce divin enfant / Lui rendre leurs hommages, // - Je suis votre vicaire, / Dit le pape en entrant, / Et même sur la terre / Votre représentant. / - Quoi, mon vicaire, toi? / Dit l'enfant en colère / Quitte donc l'or et la grandeur [...] / Jésus bon patriote, / A ces Messieurs les rois / En style sans culotte, / Prêcha beaucoup nos droits, / - Ah! Dit le roi d'Espagne, / Ah! Quel affreux blasphème! / Oh mon Dieu, mes inquisiteurs, / S'ils entendaient telles horreurs, / Vous brûleraient vous-même! [...] / Tout près de Robespierre / Joseph vit Desmoulins [... $]^{53}$.

Mais la Révolution n'est pas bien accueillie par un chacun, comme on sait: il existe aussi des noëls contre-révolutionnaires, dont voici quelques exemples: "Menons paître nos bêtes, / A la buvette accourons tous; / Menons paître nos bêtes / Dedans l'île des Fous. // Target, Fréteau / Le Mirabeau / Rhédon, Barnave et Malouet / Ne nous laisseront qu'un plat net. / Muselons bien ces bêtes, / Allons en force, accourons tous;/ Muselons bien ces bêtes / Qui nous mangeraient tous [...] // Or, écoutez, et que chacun apprenne / Que liberté vient siéger à Paris, / Et que l'on doit nous donner pour étrennes /

\footnotetext{
45 Anonyme, Or, voilà Noël passé, dans: La Grande Bible des noëls tant vieils que nouveaux, composez à la louange de Dieu et de la Vierge Marie, Troyes, chez Nicolas Oudot, 1681, éd. Poulaille (1950, pp. 353 ss.).

${ }^{46}$ H. Poulaille, ibid., pp. 35-36.

47 "La marquise de la Baume se fit connaître par ses charmes, ses caprices, son humeur quinteuse et le scandale de sa vie" (note de l'éd.).

${ }^{48}$ Noël, 1652, dans: Le Nouveau Siècle de Louis XIV (1857, p. 51).

${ }^{49}$ Noël, 1684, ibid., pp. 111-112.

${ }^{50}$ Noël, 1696, qui se chante sur l'air des Bourgeois de Chastres, dans: Poulaille (1950, pp. 37-38).

${ }^{51}$ Citoyen Th. Rousseau, A bas les rois, dans: La grande Bible des Noëls et Cantiques en l'honneur de la Liberté, Poulaille (1950, p. 585).

${ }^{52}$ Id., Ah! Que la loi nouvelle, noël républicain dédié aux membres de la Convention Nationale, dans: La grande Bible des Noëls et Cantiques en l'honneur de la Liberté, Poulaille, ibid., p. 587.

${ }^{53}$ Anonyme, Dieu se fait sans culotte, noël républicain, dans: ibid., pp. 590-535.
} 
A chacun un garde en notre logis; / C'est pour nous prendre / Sans plus attendre / Les revenus / Qui ne sont pas venus" ${ }^{54}$.

${ }^{54}$ Anonyme, Menons paître nos bêtes, noël contre-révolutionnaire, Bibliothèque Nationale, $8^{\circ} \mathrm{Ye}$, pièce 4595 , dans: ibid., pp. 595, 598.

In memoriam. Le noël commence à perdre du terrain au XIXe siècle. Une autre forme de littérature prend la relève: celle du feuilleton, qui paraît dans les journaux à bon marché, illustré au surplus, grâce à l'invention de la lithographie. Le grand public raffole désormais de cette littérature produite en série, faite d'histoires interminables de cape et d'épée (dues, d'ailleurs, plus d'une fois, à un auteur dont la renommée s'est perpétuée jusqu'à nos jours, comme Balzac, Dumas ou Sue). Le noël traditionnel, exempt de tout élément profane (de toute référence à une quelconque actualité) se retire dans les églises: là seulement retentit encore le chant d'inspiration éminemment religieuse. Mais au cours du XXe siècle, une nouvelle évolution se dessine: le noël se commercialise. En effet, partout dans le monde, pendant l'Avent, on n'entend guère plus que des chansons qui ont très peu, sinon rien à faire avec la naissance du Christ, mais plutôt avec le rêve d'un Noël sous la neige ("I'm dreaming of a white Christmas"; "Jingle Bells"), d'une agréable ambiance familiale et de la bonne bouffe - ce dont la fameuse bûche est devenue l'un des emblèmes: dessert favori du Réveillon, à vendre, au mois de décembre, dans n'importe quel supermarché de France.

\section{Bibliographie}

Aneau, Barthélémy (1539), Chant natal, contenant sept Noelz, apud Seb. Gryphius, Lugduni.

Bachelin, H. (1927), Les Noëls français, Paris.

Barbazan, E. (1976), Fabliaux et contes des poètes françois des XIe, XIIe, XIIIe, XIVe et XVe siècles, Genève (réimpr. de l'éd. de Paris, 1808).

Du Bellay, J. (1934), Euvres poétiques, vol. II, Recueil de sonnets, éd. Henri Chamard, Paris.

Fèbvre, L. et H.J. Martin (1971), L'apparition du livre, Paris.

Littré, E. (1869), Dictionnaire de la langue française, tome second, première partie, Paris.

Mâle, E. (1924), L’art religieux en France, vol. III, Paris.

Marot, Cl. (1987), L’’Adolescence Clémentine, éd. Frank Lestringant, Paris. 
Zumthor, P. (1984), La poésie de la voix dans la civilisation médiévale, Paris.

Martijn Rus

Université d'Utrecht

Trans 10

3512 JK Utrecht

martijn.rus@let.uu.nl

Navarre, M. (s.d.), Comédie de la Nativité au Moyen Age et dans les temps de JésusChrist, éd. Pierre Jourda, Paris.

Nouveau Siècle (1857), Le Nouveau Siècle de Louis XIV. Choix de Chansons (16171713), éd. Garnier Frères, Paris.

D’Ortigue, Jos. (1860), Dictionnaire liturgique, historique et théorique de plain-chant et de musique religieuse au Moyen Age et dans les temps modernes, art. Noël de A. Arnaud.

Petit Robert (1989), Dictionnaire alphabétique et analogique de la langue française, réd. A. Rey et J. Rey-Debove, Paris.

Poulaille, H. (1942), La grande et belle Bible des Noëls anciens. Du XIIe au XVIe siècle, vol. I, Paris.

Poulaille, H. (1950), La grande et belle Bible des Noëls anciens. XVIIe et XVIIIe siècles, vol. II, Paris.

De Smidt, J.H.R. (1932), Les Noëls et la tradition populaire, Amsterdam.

Vloberg, M. (1953), Les Noëls de France, Paris-Grenoble.

Von Wartburg, W. (1955), Französisches Etymologisches Wörterbuch, Basel, vol. VII. 